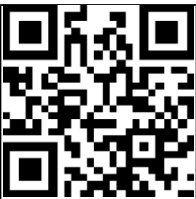

Editor's choice Scan to access mo free content

${ }^{1}$ Department of Pediatrics, Aarhus University Hospital Skejby, Aarhus, Denmark ${ }^{2}$ Department of Pediatrics, Aalborg University Hospital, Aalborg, Denmark

\section{Correspondence to} Henrik Hasle, Department of Pediatrics, Aarhus University Hospital Skejby, Palle Juul-Jensens Boulevard 99, Aarhus N 8200, Denmark; hasle@dadlnet.dk

Abstract published as a part of Pediatric Rheumatology Society Congress 2014

Received 22 October 2014 Revised 13 April 2015 Accepted 6 July 2015 Published Online First 27 July 2015

\section{SLinked}

http://dx.doi.org/10.1136/ archdischild-2015-308607

\section{CrossMark}

To cite: Brix N, Rosthøj S, Herlin T, et al. Arch Dis Child 2015;100:821-825.

\title{
Arthritis as presenting manifestation of acute lymphoblastic leukaemia in children
}

\author{
Ninna Brix, ${ }^{1}$ Steen Rosthøj, ${ }^{2}$ Troels Herlin, ${ }^{1}$ Henrik Hasle ${ }^{1}$
}

\begin{abstract}
Background At disease onset, children with acute lymphoblastic leukaemia (ALL) may present with arthralgia or even signs of arthritis. This might cause misdiagnosis and thereby lead to prolonged diagnostic delay. The present study aimed to identify children with ALL with joint involvement and to compare their characteristics and outcome with children with ALL without joint involvement.
\end{abstract}

Methods Case records of 286 children diagnosed with ALL between 1992 and 2013 were reviewed and analysed in this retrospective, descriptive study.

Results Fifty-three (18.5\%) children with ALL presented with localised joint pain, and half of them had objective signs of arthritis. The mean number of joints involved was 2.5 , most frequently presenting as asymmetric oligoarthritis. The suspected misdiagnosis were reactive arthritis (19/53), osteomyelitis (9/53) and juvenile idiopathic arthritis $(8 / 53)$. Children with joint involvement had less objective signs of haematological disease. Cytopenia was absent in 24\% in children with joint involvement (vs $8 \%$ without, $p=0.001$ ), $50 \%$ had only one cell line affected (vs $21 \%, \mathrm{p}=0.0005$ ) and $44 \%$ had no organomegaly (vs $29 \%, \mathrm{p}=0.05$ ). Median diagnostic delay was 4 vs 2 weeks. The 5 -year event-free and overall survival was superior for children with joint involvement: $94 \%$ vs $87 \%(p=0.049)$, and $96 \%$ vs $83 \%(p=0.044)$. Conclusions ALL with joint involvement is a frequent finding (18.5\%). The clinical signs of leukaemia are less prominent, but non-articular pain should alert the clinician of a possible diagnosis of leukaemia. The overall and event-free survivals were superior compared with the children without joint involvement.

\section{INTRODUCTION}

Acute lymphoblastic leukaemia (ALL) is the most common childhood neoplasia, with an annual incidence of 38-40 per million in the Western population. ${ }^{1}$ ALL has a peak incidence between 2 and 6 years of age and occurs more frequently in boys than in girls. ${ }^{2}$

Children with ALL characteristically present with fever, pallor, skin or mucosal bleeding, organomegaly and cytopenia. ${ }^{3}$ Musculoskeletal symptoms are frequent in children with ALL, usually as diffuse bone pain in the lower extremities. Some of the patients present with arthralgia (joint pain) or even with overt arthritis (localised joint swelling, redness, restriction of motion). This arthritis is generally secondary to bone, periosteal or capsular lesions rather than to direct synovial involvement. ${ }^{4-6}$ This subset of children with joint involvement may initially be misdiagnosed as reactive arthritis, osteomyelitis or juvenile idiopathic arthritis (JIA). ${ }^{7-10}$

\section{What is already known}

Paediatric acute lymphoblastic leukaemia may present with arthralgia or even signs of arthritis. Presentation of musculoskeletal complaints in a child with acute leukaemia could imply a diagnostic delay.

\section{What this study adds}

Acute lymphoblastic leukaemia with joint involvement is a frequent finding (18.5\%). At presentation, cytopenia and hepatosplenomegaly are rarely seen in ALL with joint involvement. The overall and event-free survivals were superior compared with the children without joint involvement.

In the present study, we have reviewed the clinical presentation of a large Danish cohort of children with ALL to determine the proportion with joint involvement. We recorded the clinical presentation of the patient, clinical signs of arthritis and initial rheumatological diagnosis. The aim of this study was to identify ALL patients with joint involvement, who initially were misdiagnosed as having a rheumatological disease, and further to compare their clinical and laboratory characteristics and outcome with the children with ALL without joint involvement.

\section{MATERIALS AND METHODS}

From January 1992 to March 2013 (Aarhus) and June 2012 (Aalborg), 318 children and adolescents were diagnosed with ALL at the Pediatric Oncology Units, Aarhus University Hospital Skejby $(n=219)$, and Aalborg University Hospital $(n=99)$. For the present analysis, 32 children were excluded: 5 infants aged <1 year, 12 adolescents aged $\geq 15$ years, 4 children with Down syndrome, 6 children with mature B-cell leukaemia and 5 children with missing records.

Patient records of 286 children were reviewed in this retrospective descriptive study. The following data were extracted: age, sex, symptoms recorded at the time of admission (musculoskeletal, constitutional and symptoms of cytopenia), approximate duration of symptoms, clinical signs (pallor, fever, skin or mucosal bleeding, hepatosplenomegaly, lymph node enlargement, joint swelling, redness 
and restriction of motion), laboratory results at diagnosis (complete blood count, serum lactate dehydrogenase (LDH), serum uric acid), percentage of blasts in the blood and bone marrow findings. Diffuse pain in both legs was classified as leg pains, pain distinctly localised to one or more joints as arthralgia. Presence of joint swelling was defined as arthritis, some also had redness and restriction of motion. Children with arthralgia and/ or arthritis were considered to have joint involvement. The time from onset of symptoms until diagnosis = diagnostic delay, including both patient, doctor and system delay.

Quantitative haematological data were categorised using the following definitions: leucopenia=white blood cell count $\left(\right.$ WBC) $<4.0 \times 10^{9} / \mathrm{L}$, leukocytosis $=\mathrm{WBC}>20.0 \times 10^{9} / \mathrm{L}$, neutropenia $=$ absolute neutrophil count $\left(\right.$ ANC) $<1.0 \times 10^{9} / \mathrm{L}$, anaemia $=$ haemoglobin $<10.0 \mathrm{~g} / \mathrm{dL}$ (independent of age) and thrombocytopenia $=$ platelet count $<100 \times 10^{9} / \mathrm{L}$. Upper limits for normal serum $\mathrm{LDH}$ were $500 \mathrm{IU} / \mathrm{L}$, and for serum uric acid $0.35 \mathrm{IU} / \mathrm{L}$.

Data analysis was mainly descriptive and selected differences in frequencies were tested for statistical significance using Fisher's exact test with two-sided tests and 5\% level of significance. The event-free and overall survival were determined using Kaplan-Meier plotting.

\section{RESULTS}

Of the 286 children, 243 (85\%) had pre-B ALL and 43 (15\%) had T-ALL. The cohort included 143 boys and 143 girls, comprising 188 children aged 1-5 years and 98 children aged 6-14 years. Median age was 4.4 years.

Children with arthralgia and/or arthritis were considered to have joint involvement. Of the 286 children with ALL, 53 (18.5\%) had joint involvement: all had arthralgia and 27 patients had clinical signs of arthritis. The characteristics of the children with and without arthritis are compared in table 1.

Fourteen per cent of the children reported an initial, often minor, trauma. Asymmetric oligoarthritis was most frequent; $60 \%$ had involvement of 1-2 joints, and 12\% had involvement of $>4$ joints. The most frequent joints involved were the knee (36\%) and hip (34\%). Further, 13\% each had involvement of the ankle, wrist or elbow. The shoulder and the small joint in the hands each were involved in $8 \%$. The sacroiliac joint/ spine was in involved in $6 \%$ and the small joints in the feet in $4 \%$.

Rheumatic disease was suspected initially in $68 \%$ of the 53 cases with joint involvement, with reactive arthritis as the most frequent diagnosis, 36\% $(n=19)$. Osteomyelitis and JIA were each suspected in respectively $17 \%(n=9)$ and $15 \%(n=8)$ of the children with joint involvement. The children with clinical signs of arthritis were initially diagnosed as having a rheumatic disease in $88 \%$ of cases, and the median diagnostic delay in this group was 45 days, being 17 days longer than for the children with arthralgia only.

The eight children who were suspected as JIA (median six joints involved), and six of these received rheumatological treatment: all six received NSAIDs, five also intra-articular steroid injection and one received methotrexate. This group all had negative rheumatoid factor, anti-dsDNA and antinuclear antibodies (ANA) (except one with slightly elevated ANA of eight). All had increased erythrocyte sedimentation rate and $\mathrm{C}$ reactive protein.

The group of children with and without joint involvement had similar gender distribution (49\% and 50\% boys, respectively) and age distribution (median 4.4 years of age). Children with joint involvement were all diagnosed with pre-B ALL, except one child who had T-ALL. In the group of children without joint involvement, 42 of 233 (18\%) had T-ALL. Characteristics of the two groups are compared in table 2.

The children with joint involvement had non-articular pain in the legs or spine, or had a limp more frequently $(73 \%$ vs $28 \%)$. Constitutional symptoms were less frequent $(55 \%$ vs $81 \%$ ), as were symptoms of cytopenia, especially bleeding manifestations (10\% vs $43 \%)$. The duration of symptoms also differed. The children with joint involvement initially had musculoskeletal symptoms (including joint pain) and constitutional symptoms (median duration 4 weeks), and symptoms of cytopenia appearing shortly before ALL diagnosis (median duration 1 week). The children without joint involvement initially had non-articular pain (median duration 4 weeks), then constitutional symptoms (median duration 3 weeks), and finally, symptoms of cytopenia (median duration 1 week). The total median diagnostic delay was twice as long for the children with joint involvement compared with the children without: 4 vs 2 weeks.

Table 1 Clinical characteristics of acute lymphoblastic leukaemia (ALL) children with joint involvement according to signs of arthritis

\begin{tabular}{|c|c|c|c|c|}
\hline & $\begin{array}{l}\text { Joint involvement } \\
n=53\end{array}$ & $\begin{array}{l}\text { No arthritis } \\
n=26\end{array}$ & $\begin{array}{l}\text { Arthritis } \\
\mathrm{n}=27\end{array}$ & $\mathrm{p}$ Value \\
\hline Initial trauma & $14 \%$ & $12 \%$ & $15 \%$ & 1.0 \\
\hline Nocturnal pain & $6 \%$ & $4 \%$ & $7 \%$ & 1.0 \\
\hline \multicolumn{5}{|l|}{ Number of joints involved } \\
\hline 1 & $36 \%$ & $41 \%$ & $31 \%$ & 0.39 \\
\hline 2 & $23 \%$ & $31 \%$ & $15 \%$ & 0.20 \\
\hline 3 & $19 \%$ & $16 \%$ & $23 \%$ & 0.73 \\
\hline 4 & $10 \%$ & $8 \%$ & $12 \%$ & 1 \\
\hline$>4$ & $12 \%$ & $4 \%$ & $19 \%$ & 0.19 \\
\hline Median number of joint & 2.5 & 2.1 & 2.8 & \\
\hline Total median Symptom interval (days) & 30 & 28 & 45 & \\
\hline Initial misdiagnosis & $68 \%$ & $36 \%$ & $88 \%$ & $<0.01$ \\
\hline Reactive arthritis & $36 \%$ & $31 \%$ & $45 \%$ & 0.05 \\
\hline Osteomyelitis & $17 \%$ & $5 \%$ & $19 \%$ & 0.05 \\
\hline Juvenile idiopathic arthritis & $15 \%$ & $0 \%$ & $26 \%$ & 0.01 \\
\hline
\end{tabular}

Bold type face is partly to highlight and partly to mark where there is value. 
Table 2 Comparison of the clinical characteristics of acute lymphoblastic leukaemia (ALL) children with and without joint involvement

\begin{tabular}{|c|c|c|c|}
\hline & $\begin{array}{l}\text { Joint involvement } \\
n=53, \% \text { (median diagnostic delay) }\end{array}$ & $\begin{array}{l}\text { No joint involvement } \\
\mathrm{n}=233, \% \text { (median diagnostic delay) }\end{array}$ & p Value \\
\hline Age, median & 4.4 years & 4.4 years & \\
\hline Boys & $49 \%$ & $50 \%$ & 1 \\
\hline T-ALL & $2 \%$ & $18 \%$ & $<0.01$ \\
\hline Non-articular pain & $73 \%$ (4 weeks) & $28 \%$ (4 weeks) & 0.01 \\
\hline Leg pain & $45 \%$ & $18 \%$ & $<0.01$ \\
\hline Back pain & $28 \%$ & $8 \%$ & $<0.01$ \\
\hline Limp & $22 \%$ & $18 \%$ & 0.44 \\
\hline Constitutional symptoms, total & $55 \%$ (4 weeks) & $81 \%$ (3 weeks) & $<0.01$ \\
\hline Fatigue & $45 \%$ & $64 \%$ & 0.02 \\
\hline Anorexia & $26 \%$ & $31 \%$ & 0.62 \\
\hline Abdominal pain & $29 \%$ & $22 \%$ & 0.37 \\
\hline Headache & $10 \%$ & $13 \%$ & 1 \\
\hline Anorexia & $26 \%$ & $31 \%$ & 0.62 \\
\hline Signs of cytopenia, total & $61 \%$ (1 week) & $72 \%$ (1 week) & 0.10 \\
\hline Pallor & $28 \%$ & $46 \%$ & 0.02 \\
\hline Fever & $57 \%$ & $49 \%$ & 0.36 \\
\hline Bleeding & $10 \%$ & $43 \%$ & $<0.01$ \\
\hline No signs & $24 \%$ & $8 \%$ & $<0.01$ \\
\hline Organomegaly & $56 \%$ & $71 \%$ & $<0.01$ \\
\hline Hepatomegaly & $29 \%$ & $47 \%$ & 0.01 \\
\hline Splenomegaly & $12 \%$ & $39 \%$ & $<0.01$ \\
\hline No organomegaly & $44 \%$ & $29 \%$ & 0.05 \\
\hline Lymphadenopathy & $33 \%$ & $38 \%$ & 0.64 \\
\hline Abnormal blood count & $76 \%$ & $92 \%$ & $<0.01$ \\
\hline $\mathrm{Hb}<10 \mathrm{~g} / \mathrm{dL}$ & $59 \%$ & $80 \%$ & $<0.01$ \\
\hline Platelet count $<100 \times 10^{9} / \mathrm{L}$ & $44 \%$ & $75 \%$ & $<0.01$ \\
\hline WBC $>20 \times 10^{9} / L$ & $17 \%$ & $36 \%$ & $<0.01$ \\
\hline $\mathrm{WBC}<4 \times 10^{9} / \mathrm{L}$ & $27 \%$ & $28 \%$ & 0.99 \\
\hline ANC $<1.0 \times 10^{9} / \mathrm{L}$ & $67 \%$ & $72 \%$ & 0.40 \\
\hline Lymphoblasts in blood & $70 \%$ & $75 \%$ & 0.74 \\
\hline $\mathrm{LDH}>500 \mathrm{IU} / \mathrm{L}$ & $63 \%$ & $70 \%$ & 0.33 \\
\hline Uric acid >0.5 IU/L & $17 \%$ & $30 \%$ & 0.06 \\
\hline
\end{tabular}

Bold type face is partly to highlight and partly to mark where there is value.

ANC, absolute neutrophil count; Hb, haemoglobin; LDH, lactate dehydrogenase; WBC, white blood cell count.

Organomegaly was absent more frequently in children with joint involvement $(44 \%$ vs $29 \%, \mathrm{p}=0.05)$. Enlarged lymph nodes were equally likely to be present (33\% and 38\%), but enlargement of the liver or the spleen was less frequent, and only $6 \%$ had combined lymphadenopathy and hepatosplenomegaly compared with $27 \%$ of the children without joint involvement. Similarly, the characteristic clinical triad of fever, pallor and bleeding was infrequent in children with joint involvement (6\% vs $18 \%, \mathrm{p}=0.03)$.

ALL with joint involvement had less frequently signs of cytopenia $(76 \%$ vs $92 \%, p=0.001)$ and two or three cell lines affected $(50 \%$ vs $79 \%, \mathrm{p}=0.0005)$. Anaemia, thrombocytopenia and leukocytosis were significantly less frequent in the children with ALL with joint involvement (table 2).

The survival for children with ALL with and without joint involvement is depicted in figure 1 (event-free survival, EFS) and figure 2 (overall survival). The 5-year EFS was 94\% for children with joint involvement compared with $87 \%$ for those without $(p=0.049)$. The 5 -year overall survival was superior for the group of children with joint involvement $96 \%$ vs $83 \%$ $(p=0.044)$. Restricting the survival analyses to pre-B ALL showed the same tendency of better outcome in those with joint involvement, but the difference was not statistically significant.
Of the 286 children, 97\% achieved complete remission, but $10 \%$ relapsed, $3 \%$ died in first complete remission and $1 \%$ had a secondary malignant neoplasia. The median duration of follow-up for the children alive was 9.8 years (10.1 years for the children with joint involvement and 9.7 years for the children without).

\section{DISCUSSION}

Physicians evaluating children with joint involvement must always be aware of the possibility of underlying malignancy. In this study, we investigated the extent and significance of arthralgia and/or arthritis as a presenting manifestation of ALL in children. We demonstrated a frequent occurrence of misdiagnosis and few clinical and laboratory signs of leukaemia among children with joint involvement, thus reflecting a diagnostic delay being twice as long as for the children without joint involvement.

This study describes a consecutive series of children with ALL admitted to two paediatric oncology centres in Denmark, but being retrospective the results must be interpreted with some caution and has its limitations. There is a risk of bias by parental recall as the exact duration of symptoms may be difficult to recall. Physical signs have been revealed by multiple doctors and similarly may be subject to error. The laboratory data are 


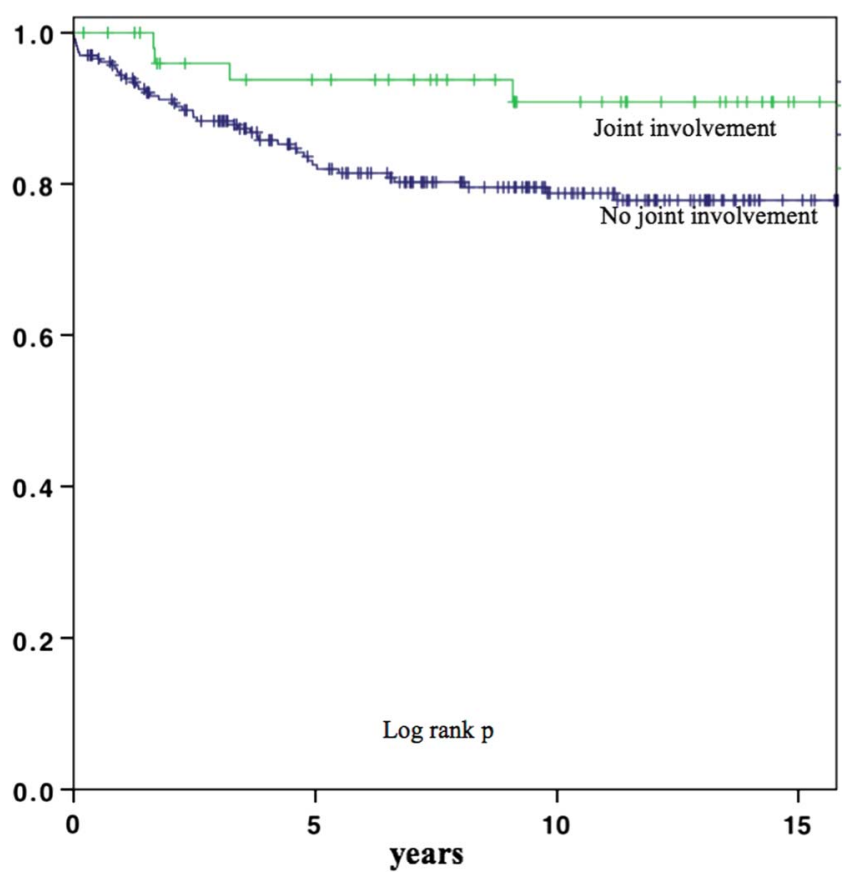

Figure 1 Event-free survival in children with acute lymphoblastic leukaemia with or without joint involvement.

accurate, but the categorical transformation is arbitrary although used previously. ${ }^{11}{ }^{12}$ When comparing the children from 1992 to 2002 with the children from 2003 to 2013 , the proportion of children with joint involvement and other musculoskeletal symptoms was unchanged as was the diagnostic delay over time. The children with T-ALL biased the survival as they were overrepresented in the group of children without joint involvement.

The present series illustrates that diagnosis of leukaemia in children with joint involvement is challenging. It is well known that bone pain, often an early symptom in ALL, is associated with nearly normal haematological counts. ${ }^{5} 13$ The clinical

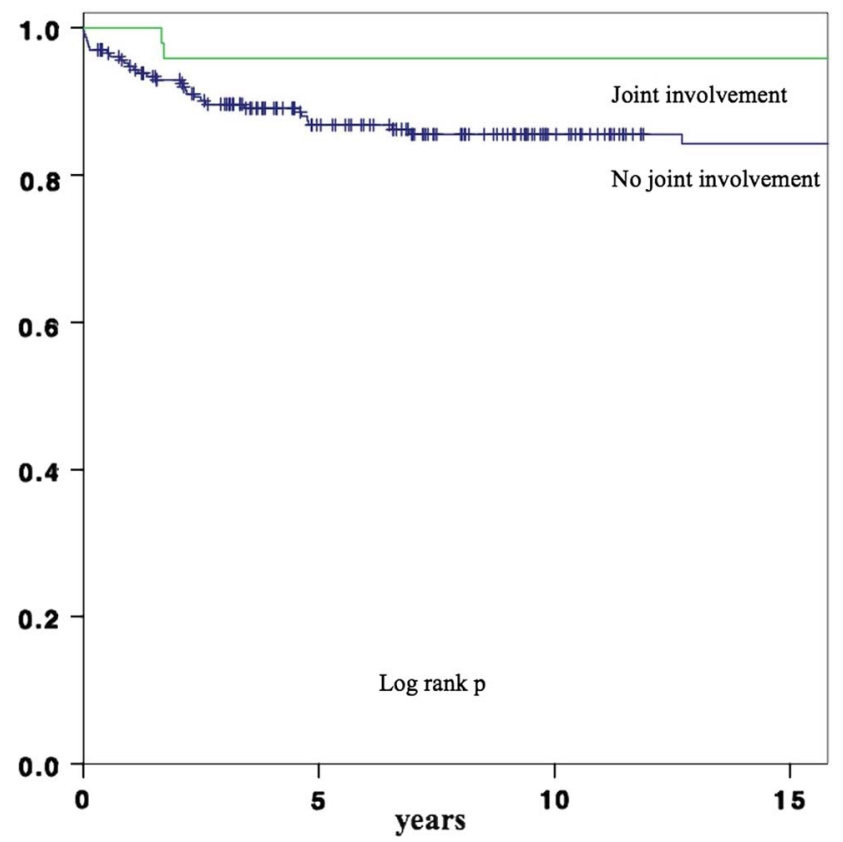

Figure 2 Overall survival in children with acute lymphoblastic leukaemia with or without joint involvement. presentation, and the consequences of isolated joint involvement, however, is sparsely described. ${ }^{14-16}$ The main focus in the literature has been the differentiation of children with musculoskeletal symptoms caused by ALL from children with

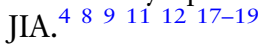

In this study, we attempted to delineate the characteristics of ALL with joint involvement, that is, pain localised to specific joints with and without objective signs of arthritis, comparing the clinical presentation and survival of children with and without joint involvement. We found that $18.5 \%$ of the children with ALL had joint involvement at time of admission, and half of them had objective signs of arthritis. An asymmetric oligoarthritis with involvement of one or two large joints was the typical presentation. Prior studies of arthralgia or arthritis in children with ALL are few. Joint pain was present at diagnosis in $16 \%$ in one study, ${ }^{13}$ whereas arthritis has been found to be present in 2-10\%. ${ }^{4} 152021$ Gur et al ${ }^{22}$ studied the rheumatic manifestations preceding adult acute leukaemia and found that $3.6 \%$ presented as a reactive arthritis with asymmetric involvement of the large joints. In our series of children with ALL, $6.7 \%$ had an initial presentation mimicking reactive arthritis, 3\% as osteomyelitis and $2.7 \%$ as JIA. This is slightly different from previous literature stating that $2-3.6 \%$ presented as reactive arthritis, $1.5 \%$ as osteomyelitis ${ }^{162223}$ and $6.4 \%$ presented as JIA. ${ }^{6}$

The risk of misdiagnosis is increased by the relative lack of clinical and laboratory signs of leukaemia. Half of the children with joint involvement had less than two haematological cell lines affected, 24\% did not have any signs of cytopenia, 44\% did not have any organomegaly or lymph node enlargement. This may have an impact on diagnostic delay. We found that the diagnostic delay was twice as long in children with joint involvement as in those without. The atypical presentation among children with musculoskeletal involvement has been suggested to prolong the diagnostic delay in previous reports. ${ }^{5} 2124-26$ The present study indicates the same tendency among children with joint involvement. This underscores the need for prompt and early investigations to identify the ALL patients among children with joint-related symptoms and signs of arthritis.

The clinical picture gets further complicated if glucocorticosteroids are administrated since this affects blood counts and bone marrow. ${ }^{14} 1527$ Subtle signs of leukaemia or indications for steroid treatment should prompt considering a bone marrow examination.

Marwaha et $a l^{6}$ stated that $6.4 \%$ of 762 children with ALL had an initial presentation mimicking JIA. This study and Ostrov et $a l^{9}$ stated that the children with ALL had painful, transient and asymmetric oligoarthritis and like in our study general physical examination for signs of leukaemia often was not useful. Though in our study $73 \%$ of the children with joint involvement also had non-articular pain at presentation, and while articular pain is a very common rheumatic complaint, non-articular musculoskeletal pain or bone tenderness should cause suspicion of leukaemia. A smaller Indian study from $2009^{15}$ found that non-articular pain predicted a diagnosis of ALL and differentiated it from JIA.

Serum LDH is a marker of high cell turnover and was elevated among the majority (63\%) of the children with joint involvement. In previous studies, the majority of children with ALL and musculoskeletal involvement did not have lymphoblasts in the peripheral blood at the time of clinical evaluation. ${ }^{11}{ }^{13}$ Jones et $a l^{11}$ found that only $25 \%$ of children with ALL who presented to rheumatologists for arthritis had blasts in the peripheral blood at the time of evaluation. In our cohort, $70 \%$ of the children with ALL with joint involvement had blasts 
in peripheral blood at admission equivalent to the children with ALL without joint involvement. This discrepancy might be a consequence of disease at different stages. A change of methodology over time might also explain the difference, as flow cytometry is more sensitive to blasts than morphology.

Non-articular pain, blasts in peripheral blood and elevated LDH are predictive factors for ALL in this group as they occurred among the majority of children with joint involvement. At least one of these markers was positive among $94 \%$ of the children with joint involvement.

Polyarticular arthritis, soft tissue swelling, osteopenia and normal blood counts have been shown to be more frequent among children with JIA than among children with ALL with musculoskeletal symptoms caused by ALL. ${ }^{6}{ }^{12}$ Each type of arthritis, as well as osteomyelitis, have different clinical characteristics, that is, age at presentation, sex ratio, joint distribution and other associated features. JIA is a diagnosis of exclusion and requires the presence of arthritis for at least six weeks. ${ }^{28}$ Caution should be given to each specific diagnosis considering whether any features are atypical. ANA are not specific for rheumatic disease and may be positive in up to $30 \%$ of children hospitalised for various reasons. ${ }^{9} 1116$

The prognostic significance of musculoskeletal symptoms in ALL has been debated. Previous studies have shown that the children with severe objective skeletal abnormalities may have an indolent form of leukaemia. ${ }^{29-31}$ In the present study, we found that children with joint involvement had better event-free and overall survival, both differences reaching statistical significance. ALL with arthritis may belong to a subgroup with a better prognosis. The comparison, however, was biased by the children with T-ALL, all of whom except one were in the group without joint involvement. These children are known to have a poorer outcome, although there has been a significant improvement in survival of children with T-ALL in the two last Nordic trials. ${ }^{2}$ Restricting the survival analysis to children with pre-B ALL, the survival benefits were not statistically significant. This, however, may be due to small numbers. Thus, children with joint involvement may have a favourable prognosis in spite of the longer diagnostic delay, but even so reduction of the diagnostic delay is important to maintain the confidence and trust of children and parents and to improve the clinical conditions of the children at the time of starting therapy.

\section{CONCLUSIONS}

A high proportion of children with ALL present with joint involvement, and the risk of an initial rheumatological misdiagnose is significant. The clinical signs of leukaemia are less prominent in this group compared with the children without joint involvement. These diagnostic challenges can be minimised if clinicians are aware of the atypical presentation and pay close attention to non-articular pain, elevated LDH and blasts in peripheral blood indicating ALL. This group of children with ALL with joint involvement may belong to a subgroup with a better prognosis.

Contributors NB conceptualised and designed the study and collected the data, drafted the initial manuscript and approved the final manuscript as submitted. $\mathrm{HH}$ and TH supervised data collection, critically reviewed and revised the manuscript and approved the final manuscript as submitted. SR critically reviewed and revised the manuscript, and approved the final manuscript as submitted.

Competing interests None declared.

Ethics approval Danish Ethical Committee.

Provenance and peer review Not commissioned; externally peer reviewed.

\section{REFERENCES}

1 Hjalgrim LL, Rostgaard K, Schmiegelow K, et al. Age- and sex-specific incidence of childhood leukemia by immunophenotype in the nordic countries. J Natl Cancer Inst 2003;95:1539-44.

2 Schmiegelow K, Forestier E, Hellebostad M, et al. Long-term results of NOPHO ALL-92 and ALL-2000 studies of childhood acute lymphoblastic leukaemia. Leukemia 2010;24:345-54.

3 Riccio I, Marcarelli M, Del Regno N, et al. Musculoskeletal problems in pediatric acute leukemia. J Pediatr Orthop 2013;22:264-9.

4 Tafaghodi F, Aghighi Y, Rokni Y. et al. Predictive plain X-ray findings in distinguishing early stage acute lymphoblastic leukemia from juvenile idiopathic arthritis. Clin Rheumatol, 2009;28:1253-8.

5 Jonsson OG, Sartain P, Ducore JM, et al. Bone pain as an initial symptom of childhood acute lymphoblastic leukemia: association with nearly normal hematologic indexes. J Pediatr 1990;117:233-7.

6 Marwaha RK, Kulkarni KP, Bansal D, et al. Acute lymphoblastic leukemia masquerading as juvenile rheumatoid arthritis: diagnostic pitfall and association with survival. Ann Hematol 2010;89:249-54.

7 Schaller J. Arthritis as a presenting manifestation of malignancy in children. J Pediatr 1972;81:793-7.

8 Cabral DA, Tucker LB. Malignancies in children who initially present with rheumatic complaints. J Pediatr 1999;134:53-7.

9 Ostrov BE, Goldsmith DP, Athreya BH. Differentiation of systemic juvenile rheumatoid arthritis from acute leukemia near the onset of disease. J Pediatr 1993;122:595-8.

10 Bradlow A, Barton C. Arthritic presentation of childhood leukaemia. Postgrad Med $J$ 1991;67:562-4.

11 Jones OY, Spencer CH, Bowyer SL, et al. A multicenter case-control study on predictive factors distinguishing childhood leukemia from juvenile rheumatoid arthritis. Pediatrics 2006;117:e840-4.

12 Tamashiro M. Discrimination of acute lymphoblastic leukemia from systemic-onset juvenile idiopathic arthritis at disease onset. Clinics (Sao Paulo) 2011;66:1665-9.

13 Ma SK, Chan GC, Ha SY, et al. Clinical presentation, hematologic features and treatment outcome of childhood acute lymphoblastic leukemia: a review of 73 cases in Hong Kong. Hematol Oncol 1997;15:141-9.

14 Suri D, Ahluwalia J, Sachdeva MUS, et al. Arthritic presentation of childhood malignancy: beware of normal blood counts. Rheumatol Int 2011;31:827-9.

15 Gupta D, Singh S, Suri D, et al. Arthritic presentation of acute leukemia in children: experience from a tertiary care centre in North India. Rheumatol Int 2010;30:767-70.

16 Malleson PN, Sailer M, Mackinnon MJ. Usefulness of antinuclear antibody testing to screen for rheumatic diseases. Arch Dis Child 1997;77:299-304.

17 Trapani S, Grisolia F, Simonini G, et al. Incidence of occult cancer in children presenting with musculoskeletal symptoms: a 10-year survey in a pediatric rheumatology unit. Semin Arthritis Rheum 2000;29:348-59.

18 Robazzi TCMV, Barreto JHS, Silva LR, et al. Osteoarticular manifestations as initial presentation of acute leukemias in children and adolescents in Bahia, Brazil. J Pediatr Hematol Off J Am Soc Pediatr Hematol 2007:29:622-6.

19 Needleman M. Childhood leukaemia mimicking arthritis. J Am Board Fam Pr 1996;9(1):56.

20 Sinigaglia R, Gigante C, Bisinella G, et al. Musculoskeletal manifestations in pediatric acute leukemia. J Pediatr Orthop 2008;28:20-8.

21 Biswas S, Chakrabarti S, Chakraborty J, et al. Childhood acute leukemia in West Bengal, India with an emphasis on uncommon clinical features. Asian Pac J Cancer Prev 2009;10:903-6.

22 Gur H, Koren V, Ehrenfeld M, et al. Rheumatic manifestations preceding adult acute leukemia: characteristics and implication in course and prognosis. Acta Haematol 1999;101:1-6.

23 Riccio I, Marcarelli M, Del Regno N, et al. Musculoskeletal problems in pediatric acute leukemia. J Pediatr Orthop 2013;22:264-9.

24 Bernbeck B, Wüller D, Janssen G, et al. Symptoms of childhood acute lymphoblastic leukemia: red flags to recognize leukemia in daily practice. Klin Padiatr 2012;221:369-73.

25 Hutter JJ. Childhood leukemia. Pediatr Rev 2010:31:234-41.

26 Chell J, Fernandes JA, Bell MJ. Leukaemia in childhood. Ann R Coll Surg Engl 2000;83:186-9.

27 Révész $\mathrm{T}$, Kardos $\mathrm{G}$, Kajtár $\mathrm{P}$, et al. The adverse effect of prolonged prednisolone pretreatment in children with acute lymphoblastic leukemia. Cancer 1985;55:1637-40.

28 Rashid AKMM, Al Mamun A, Ahasan HAMN, et al. Leukaemia mimicking juvenile rheumatoid arthritis. Pak J Med Sci 2005;21:85-7.

29 Müller $\mathrm{HL}$, Horwitz $\mathrm{AE}$, Kühl MD. Acute Lymphoblastic leukemia with severe skeletal involvement: A Subset of Childhood Leukemia with a Good Prognosis Patients and Skeletal Surveys. Pediatr Hematol Oncol 1998;15:121-33.

30 Stephen D, Heinrich MS, Gallagher D, et al. The prognostic significance of the skeletal manifestaions of acute lymphoblastic leukemia of childhood. J Pediatr Orthop 1994;14:105-11.

31 Kai T, Ishii E, Matsuzaki A, et al. Clinical and prognostic implications of bone lesions in childhood leukemia at diagnosis. Leuk Lymphoma 1996;23:119-23. 